\title{
A!
}

This is an electronic reprint of the original article.

This reprint may differ from the original in pagination and typographic detail.

Castillo, Daniel; Al-Qadi, Imad

\section{Mechanical Modelling of Asphalt Concrete Using Grid Division}

Published in:

INTERNATIONAL JOURNAL OF PAVEMENT ENGINEERING

DOI:

$10.1080 / 10298436.2019 .1650926$

Published: 02/07/2020

Document Version

Peer reviewed version

Please cite the original version:

Castillo, D., \& Al-Qadi, I. (2020). Mechanical Modelling of Asphalt Concrete Using Grid Division.

INTERNATIONAL JOURNAL OF PAVEMENT ENGINEERING, 21(8), 1012-1023.

https://doi.org/10.1080/10298436.2019.1650926

This material is protected by copyright and other intellectual property rights, and duplication or sale of all or part of any of the repository collections is not permitted, except that material may be duplicated by you for your research use or educational purposes in electronic or print form. You must obtain permission for any other use. Electronic or print copies may not be offered, whether for sale or otherwise to anyone who is not an authorised user. 


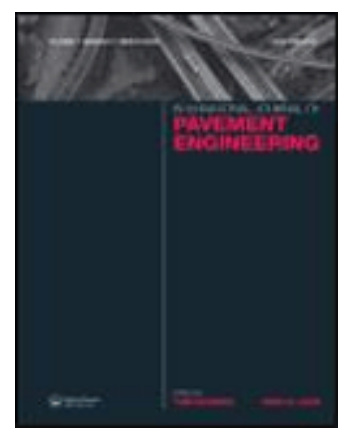

\section{Mechanical Modelling of Asphalt Concrete Using Grid Division}

\begin{tabular}{|r|l|}
\hline Journal: & International Journal of Pavement Engineering \\
\hline Manuscript ID & Draft \\
\hline Manuscript Type: & Original Article \\
\hline Keywords: & $\begin{array}{l}\text { multiphase material, modelling, grid division, finite elements, } \\
\text { microstructure }\end{array}$ \\
\hline
\end{tabular}

\section{SCHOLARONE \\ Manuscripts}




\section{MECHANICAL MODELLING OF ASPHALT CONCRETE USING GRID DIVISION}

2

3 Abstract: In this paper, a simple method is introduced for the computational modelling of

4 multiphase materials, and for the approximation of their mechanical response. The two-

5 dimensional microstructures of six asphalt concrete specimens are selected; three of the

6 specimens have 'low'-, and three have 'high' aggregate fraction. A grid is used to divide the

7 surface of each microstructure into square cells. The procedure of grid division is applied from

81 up to 100 divisions per side (i.e., up to 10,000 cells in the grid-divided specimen). To obtain

9 an approximation of the mechanical response of the microstructure, the properties of the cells

10 are estimated using three simple interpolation rules between the properties of the two phases,

11 i.e. asphalt matrix and rock aggregates. It is found that the interpolation rules can yield

12 reasonably representative results depending on the aggregate fraction of the microstructures

13 and the number of divisions/cells in the grid. The grid-divided specimens allow approximating

14 the overall mechanical response of the microstructures, and characteristics such as strain

15 concentrations, overall deformations, and resulting force.

16

17 Keywords: multiphase material, modelling, grid division, finite elements. 


\section{INTRODUCTION}

20 The process of modelling multiphase materials in two and three dimensions can become quite complex, depending on the accuracy desired in the geometric and mechanical description of the individual phases. Commonly, a modeling process requires laboratory resource-and computational cost-intensive efforts. This process may entail acquiring the geometry of multiple phases composing the material, i.e. through imaging or X-ray analysis of cored specimens (Masad et al. 1999, Gruber et al. 2012, Obaidat et al. 2017), as well as laboratory testing of individual phases to quantify their associated mechanical or physical characteristics of interest, such as moisture/thermal diffusivity, stiffness, surface free energy or linear 28 viscoelastic parameters (Kim et al. 2005, Kim et al. 2008, Yokohama and Nakashima, 2005, 29 Witthüser et al. 2006). The process may also involve the formulation or usage of heavy, timeconsuming computational models, that need calibration to yield representative results.

31 Understandably, such a process necessitates a trade-off between result accuracy and 32 computational costs. Hence, alternatives to de-escalate the complexity of the modelling process 33 should be considered, geared towards time- and cost-efficient models that consume fewer 34 resources.

35 In this context, there is interest in the area of pavements to adequately characterize the 36 geometry and mechanical behavior of the multi-phase materials that compose the pavement 37 structures, as well as numerous variables that simultaneously affect their properties, such as 38 temperature, moisture, stress regime and oxidation. To this end, complex models have been 39 developed by several researchers to represent pavement materials and structures. Many of these 40 models use the finite element (FE) method and focus particularly on the behavior of asphalt 41 concrete (AC) mixtures. Such models have been developed to predict damage and visco-plastic 42 behavior of material under loading (Darabi et al. 2011), aging (Rahmani et al. 2017), and 43 fracture using the cohesive-zone theory (Aragão et al. 2010). Tire-pavement interaction has 
44 also been considered, using measured three-dimensional contact stresses (Wang and Al-Qadi, 2009), including various tire configurations such as wide-base tires, and considering non-linear anisotropy in the lower layers of the pavement structure (Hernandez et al., 2016). In addition, the impact of uncertainty and variability on AC behavior has been studied, through random correlated spatial variability and the random generation of micromechanical configurations, as well as the usage of domain analysis methods to quantify 'bulk' damage potential (Castillo and Al-Qadi, 2018; Caro et al., 2018; Gamez et al. 2018).

These models take advantage of the versatility of the FE method to provide a representative spatial discretization of the domain of interest, and to accurately consider appropriate constitutive behaviors of the composing phases of the materials or the layers of the structure. The computational models are based on previous validations of FE predictions and provide good matches with experimental results; for instance, using viscoelastic approximations for the behavior of the AC material has proved useful for modeling distresses, such as rutting (Elseifi et al. 2006). This constitutive model was also used to predict strains in full-depth flexible pavement sections under moving loads (Wang and Al-Qadi, 2008).

In the context of the FE model, the domain of interest (i.e. the multi-phase geometry) commonly needs a finer discretization around irregularities in the material, near which critical concentrations of stresses and strains are expected to develop. Moreover, the need to consider thousands of irregularly-shape aggregates and air voids is a shortcoming. The presence of these numerous irregularities and inclusions is responsible for a significant part of the computational cost of the modeling process; characteristics such as the low thickness of the asphalt matrix between highly angular aggregates, or a high density of air voids over a small area, are directly responsible for inducing finer-than-average meshes. Considering these characteristics results not only in a greater number of finite elements, but it also hinders the meshing process and may result in non-suitable element shapes that compromise the results (i.e. leading to ill-conditioned 
matrices in the FE model). Moreover, the modelling of pre-existing cracks within the material, as well as additional parameters inherent to the model such as interaction laws between the phases, add to the complexity and associated computational cost of the models.

An alternative that has been used to address these challenges is multiscale modeling. A multiscale model alternates between a local, heterogeneous representation of the microstructure of the material, and a global, statistically homogeneous model of a structure. The scales are coupled two-way and linked through homogenization; multiscale models can include nonlinear behavior and fracture, and significantly reduce computational times using parallelization techniques (Kim et al. 2013). The local representation should be large enough to be representative of the global behavior of the mixture, but still small enough to be computationally efficient. This smallest practical unit is called a representative volume element (RVE); for instance, an RVE of $60 \mathrm{~mm}$ was found for a Superpave mixture with $12.5 \mathrm{~mm}$ nominal maximum aggregate size (NMAS) using digital image correlation (Kim et al. 2010), and in general, statistical homogeneity in the fraction of inclusions is observed from sizes greater than 2-3 times the NMAS, at least in two dimensions (Ozer et al. 2016) . Multiscale experimental data shows different levels of sensitivity depending on the scale (Underwood and Kim, 2013), through temperature and frequency sweep tests at the scales of binder/mastic, fine aggregate matrix, and asphalt mixtures.

While the approach presented herein is not multiscale, it certainly shares many of the basic characteristics of multiscale modeling. These include discretizing phases at different scales, the search for a scale/size that is representative of the actual overall response, homogenizing mechanical/physical properties of the material, and in general, offering a computationally efficient alternative to model phenomena over a highly heterogeneous domain. The main difference between the two approaches is the presence of a range of scales (multiscale modeling uses 'leaps' between scales, whereas in the presented approach the range is 
continuous), and therefore the lack of feedback between scales.

This paper offers an intermediate approach, half-way between micromechanics and

continuum-damage based modeling, to approximate the response of two-dimensional

97 specimens of AC. By discretizing their phases (aggregates and matrix) and estimating 98 mechanical properties over a grid, the complexity of the model is reduced without neglecting 99 the presence and effects of irregularities and inclusions in the domain.

100

101

102

103 concrete $(\mathrm{AC})$ microstructure:

104 - Step 1: Superimpose a grid on the surface of the microstructure, to divide it into cells.

- Step 2: Determine the aggregate fraction per cell, i.e. the percentage area of the cell that is covered by the aggregate phase.

- Step 3: Estimate the mechanical properties cell-wise, interpolating between the properties

110 Three alternatives are tested to estimate the mechanical properties of the cells in Step 3 (see

111 Section 2.3), which involve simple interpolation rules between the properties of the two 'pure'

112 phases; rock aggregates and matrix. Herein, the aggregate fraction was selected as the

113 determining parameter of mechanical behavior, since the (instantaneous) stiffness of the coarse 114 aggregates can be two or three orders of magnitude greater than that of the binder. The steps 115 are described next.

116

117 2.1. Division of the microstructure into cells, using a grid (Step 1)

118 2.1.1. Selection and mechanical properties of the AC specimens 
119 The objective of the first step is to partition the surface of the specimen into cells. A 'specimen'

120 is understood as a microstructure of $\mathrm{AC}$, i.e. a two-dimensional configuration of aggregate

121 particles embedded in a homogeneous matrix of fine aggregates, asphalt binder and air. For

122 simplicity, in this study the specimens are composed of two phases only, namely coarse

123 aggregates and asphalt matrix. For simplicity, in this application the phases are not susceptible

124 to damage, i.e. there is no reduction in the ability to sustain stresses.

125 Two values of aggregate fraction are considered in this study: a 'low' aggregate fraction of $12652.7 \%$, and a 'high' aggregate fraction of $66.1 \%$. The values of aggregate fraction tend to vary 127 over a relatively wide range. The selected values are within the range of two sources (You et 128 al., 2009, Zelelew and Papagiannakis, 2011). For each aggregate fraction, three AC 129 microstructures were randomly generated, for a total of six microstructures of AC. Two values 130 of aggregate fraction are used because the aggregate phase is expected to have a major 131 influence in the mechanical response of the microstructures, due to the high stiffness of the 132 rocks. The microstructures are squares with $100-\mathrm{mm}$ sides; the shape and size were arbitrarily 133 selected, to be within the scale of laboratory specimens. The microstructures are presented in 134 Figure 1. 


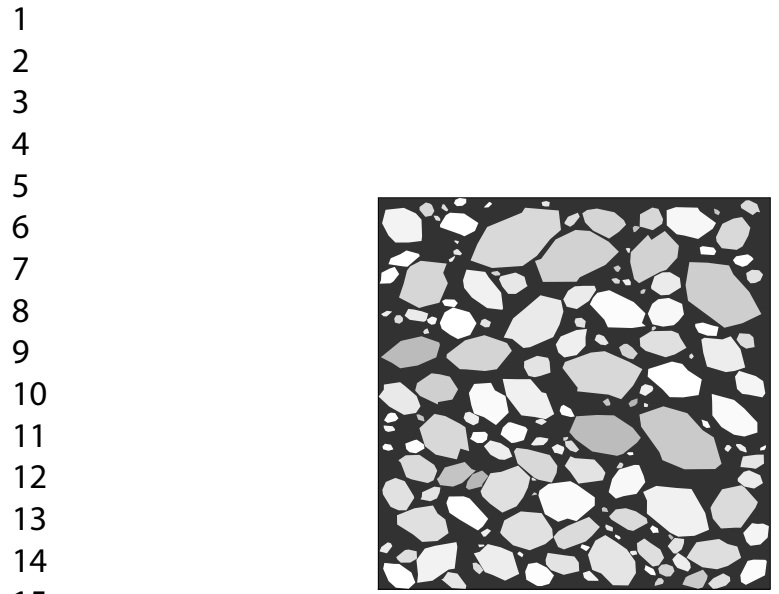

15

16

17

18

19

20

21

22

23

24

25

26

27

28

29

30

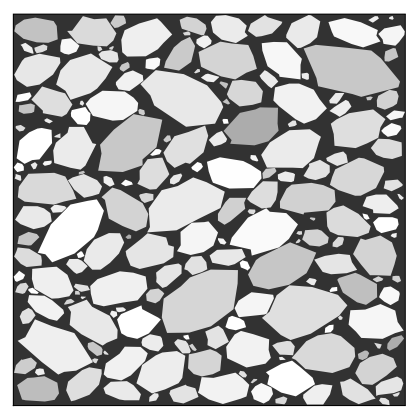

H1. $66.10 \%$

$100 \mathrm{~mm}$

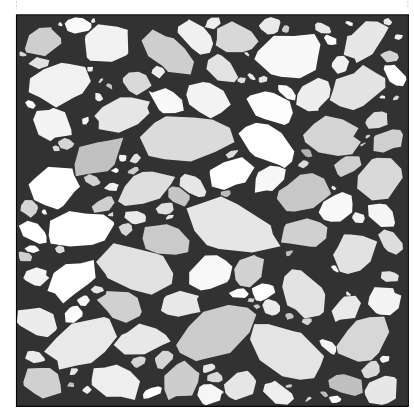

L2. $52.67 \%$

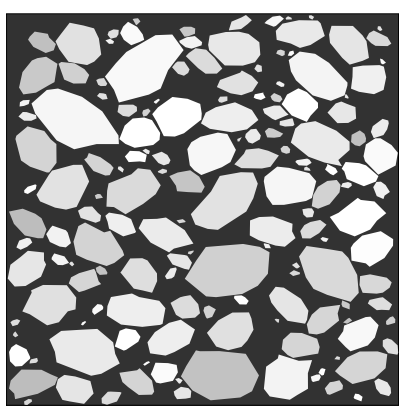

L3. $52.70 \%$

(a) Microstructures with 'Low' aggregate fraction, 52.7\%

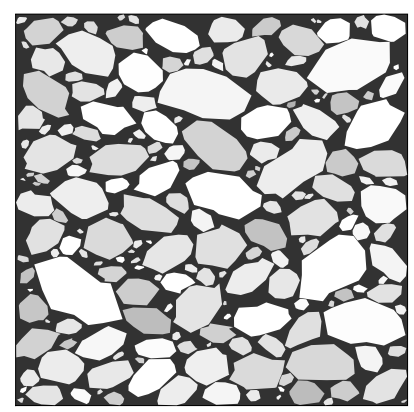

H2. $66.11 \%$

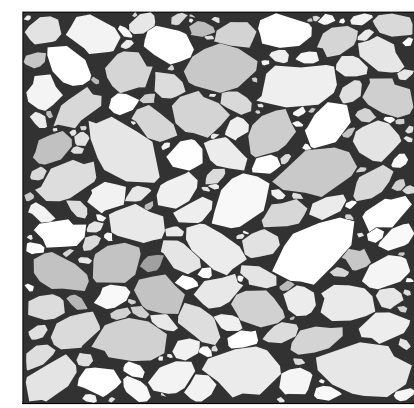

H3. $66.11 \%$

(b) Microstructures with 'High' aggregate fraction, 66.1\%

136 Figure 1. AC microstructures selected for the grid division and modelling approximation. (a)

137 Low aggregate fraction, $52.7 \%$. (b) Higher aggregate fraction, $66.1 \%$.

139 The AC microstructures were created randomly, using a MATLAB code first presented by

140 Castillo et al. (2015) and now in its latest version (MG, microstructure generator). In this study,

141 a microstructure is understood as a random spatial configuration of polygons with aggregate-

142 like characteristics, which are placed within a domain with arbitrary shape. Prescribed criteria

143 can be followed, such as probabilistic rotation and/or location of the particles. The

144 microstructures are created in two dimensions, using an algorithm to create the shapes of the

145 aggregates (i.e. without using an aggregate database). Criteria used include a random number

146 of vertices for the aggregates, random distances from each vertex to the center of the aggregate, 
147 and random elongation. The MG is capable of achieving various levels of aggregate angularity

148 and microstructure forms (Castillo et al. 2015).

149 The objective of having three microstructures for each aggregate fraction (i.e., replicates) is

150 to assess the consistency of the results; in other words, to observe if the behavior presented by

151 any of the specimens is representative of their corresponding aggregate fraction. A key for this

152 assessment is consistency among microstructures, i.e. gradation of the particles is the same for

153 all microstructures, and the two levels of aggregate fraction are kept almost constant among

154 specimens. Herein, consistency was achieved by the procedure of random generation of the

155 microstructures provided by the MG. The aggregate size of the microstructures presented in

156 Figure 1 ranges from $1.18 \mathrm{~mm}$ (sieve \#16) to $19 \mathrm{~mm}$ (sieve 3/4'”).

\subsubsection{Grid pattern}

159 Figure 2 presents three possible 'tiling patterns,' or ways to divide a two-dimensional domain

160 using a grid of cells with the same size and/or shape; squares, equilateral triangles, and regular

161 hexagons. As observed, there are many ways of dividing a domain into cells; consider that the

162 cells could also be combined to create arrays of cells of the same shape and different size.

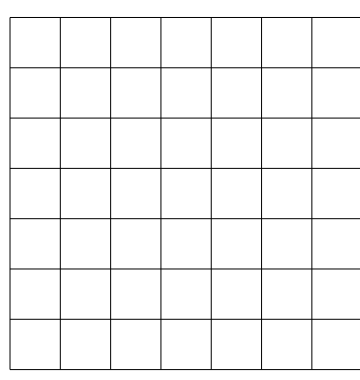

(a)

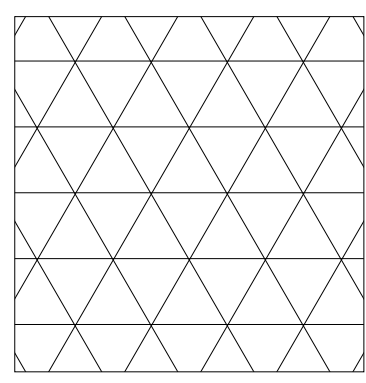

(b)

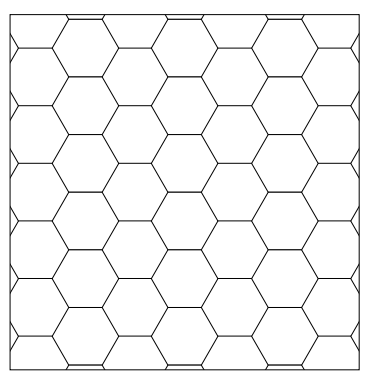

(c)

164 Figure 2. Three possible ways to divide a plane using a regular grid. (a) Square cells, (b)

165 Triangular cells, (c) Hexagonal cells.

166 
167 Although any tiling pattern could be used to divide the specimens, in this study the

168 microstructures are divided into square cells of equal size, which is perhaps the simplest way

169 to divide a plane and the most computationally favorable. Using this pattern, the grid resolution

170 can easily be doubled, which simplifies increasing the divisions (i.e. the 'next' grid with more

171 divisions is relatively easy to produce). Also, all even- and odd- divided specimens will always

172 have some nodes in the same position.

173

174 2.2. Cell-wise determination of the aggregate fraction (Step 2)

175 The six microstructures were divided using grids, in the (full) range from 1 to 100 divisions,

176 i.e. partitioning them into 1 to 10,000 cells. Selected divisions corresponding to microstructures

177 L1 and H1 from Figure 1 are presented in Figure 3. 
(a) 'Low' aggregate fraction, 52.7\% (L1 in Fig.1)

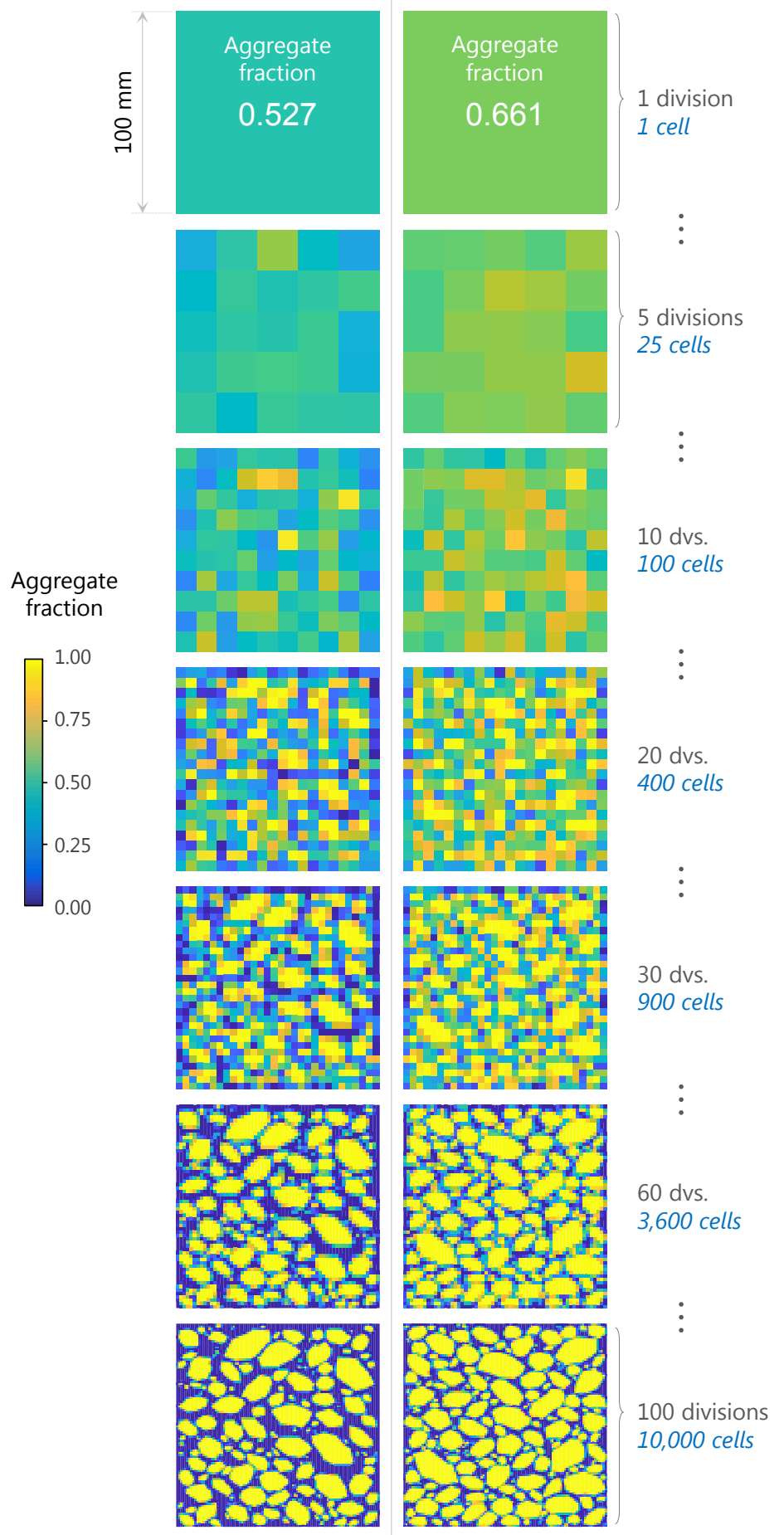

(b) 'High' aggregate fraction, 66.1\%. (H1 in Fig.1)
179 Figure 3. Grid division and aggregate fraction of (a) microstructure with low aggregate fraction

180 ('L1' from Figure 1), and (b) microstructure with high aggregate fraction ('H1' from Figure

181 1). The colors represent the percentage area of each cell that is covered with aggregates. 
183 Notice that quite early in the division process, some cells are already covered completely by 184 aggregates, and for higher numbers of divisions (or microstructures with low aggregate 185 fraction) there are also cells that comprise only asphalt matrix. These cells may prove critical 186 to the overall response of the specimen, since they will perform purely as aggregates or matrix, 187 without any kind of interpolation.

188 The grid-divided specimens from Figure 3 can be interpreted as representations of the 189 microstructures at different 'resolutions' (scales), ranging from very coarse (low resolution, 190 less than 10-20 divisions of a 100-mm side) to detailed (about 30-40 divisions and upwards, 191 for the scale used in this study). The maximum number of divisions in the grid (100) was 192 selected arbitrarily; as the number of divisions increases, the original aggregate microstructure 193 becomes more discernible.

194 Because of the complex geometry of the aggregates, calculating the aggregate fraction per 195 cell can be challenging. The uniform grid frequently isolates highly irregular partitions of 196 aggregates and matrix, and these areas become difficult to account for. Additionally, the 197 increased number of cells is a challenge, even for a specimen with relatively low divisions. In 198 this study, an automated meshing procedure was devised using (1) custom Python scripts and 199 (2) the built-in meshing algorithm from the general-purpose FE software Abaqus, as follows.

200 The matrix phase of the specimens was first isolated and partitioned using the grids, and the 201 resulting (partitioned) matrix phase was meshed with finite elements. The presence of the 202 partitions guaranteed that (1) no finite element belonged to more than one cell, and (2) the full 203 domain of the matrix phase was covered with elements. The area of the elements within each 204 cell was then accumulated (matrix area per cell), and the aggregate area per cell was obtained 205 by subtraction. 
$208 \quad$ 2.3. Interpolation rules to estimate the mechanical properties of the cells (Step 3)

209 The data resulting from the grid division (Step 2) was used as part of a simplified methodology

210 to approximate the behavior of the microstructures. Considering that the aggregate fraction per

211 cell is known for all (600) grid-divided specimens, an estimate of the mechanical properties of

212 the cells was calculated based on the elastic and linear viscoelastic properties of the 'pure'

213 aggregate and matrix phases, respectively, following three interpolation rules.

214 The aggregate phase was considered to be fully elastic, with a Young's modulus of 25 GPa

215 and a Poisson's ratio of 0.16 . These values are within the ranges reported for limestone

216 (Shakiba et al. 2015, Yasar et al. 2004, Hart and Wang, 1995). On the other hand, the matrix

217 phase was considered linear viscoelastic, with instantaneous relaxation modulus $\left(E_{o}\right)$ equal to

$218112 \mathrm{MPa}$ at $25^{\circ} \mathrm{C}$, Poisson's ratio 0.40, and Prony parameters reported by Kim et al. (2005).

219 The parameters are presented in Table 1. The same set of relaxation parameters is applied to 220 all viscoelastic cells of material, in normalized form.

221

222 Table 1. Viscoelastic mechanical parameters of the asphalt matrix, from Kim et al. (2005).

\begin{tabular}{llll}
\hline$E_{1}[\mathrm{kPa}]$ & 73,173 & $\eta_{1}[\mathrm{kPa} \cdot \mathrm{s}]$ & 115 \\
$E_{2}$ & 25,506 & $\eta_{2}$ & 418 \\
$E_{3}$ & 10,847 & $\eta_{3}$ & 1,079 \\
$E_{4}$ & 2,078 & $\eta_{4}$ & 1,529 \\
$E_{5}$ & 332 & $\eta_{5}$ & 2,249 \\
$E_{6}$ & 53.4 & $\eta_{6}$ & 3,513 \\
$E_{7}$ & 8.44 & $\eta_{7}$ & 5,430 \\
$E_{8}$ & 1.33 & $\eta_{8}$ & 8,664 \\
$E_{9}$ & 0.252 & $\eta_{9}$ & 20,406 \\
\hline$E_{\infty}$ & 0.122 & & \\
$v$ & 0.40 & & \\
\hline
\end{tabular}

224 In any of the three interpolation rules, a cell with $100 \%$ aggregate fraction will behave as a 
225 homogeneous block of rock (elastic). Conversely, a cell with $0 \%$ aggregate fraction is modelled 226 as a block of matrix (linear viscoelastic). For all other cells with aggregate fractions in the 0$227100 \%$ range, three simple interpolation rules between the two phases are tested. An example 228 showcasing the three alternatives is presented in Figure 4 for a grid-divided specimen with 40 229 divisions, i.e. 1,600 cells (microstructure 'L2' in Figure 1).

230

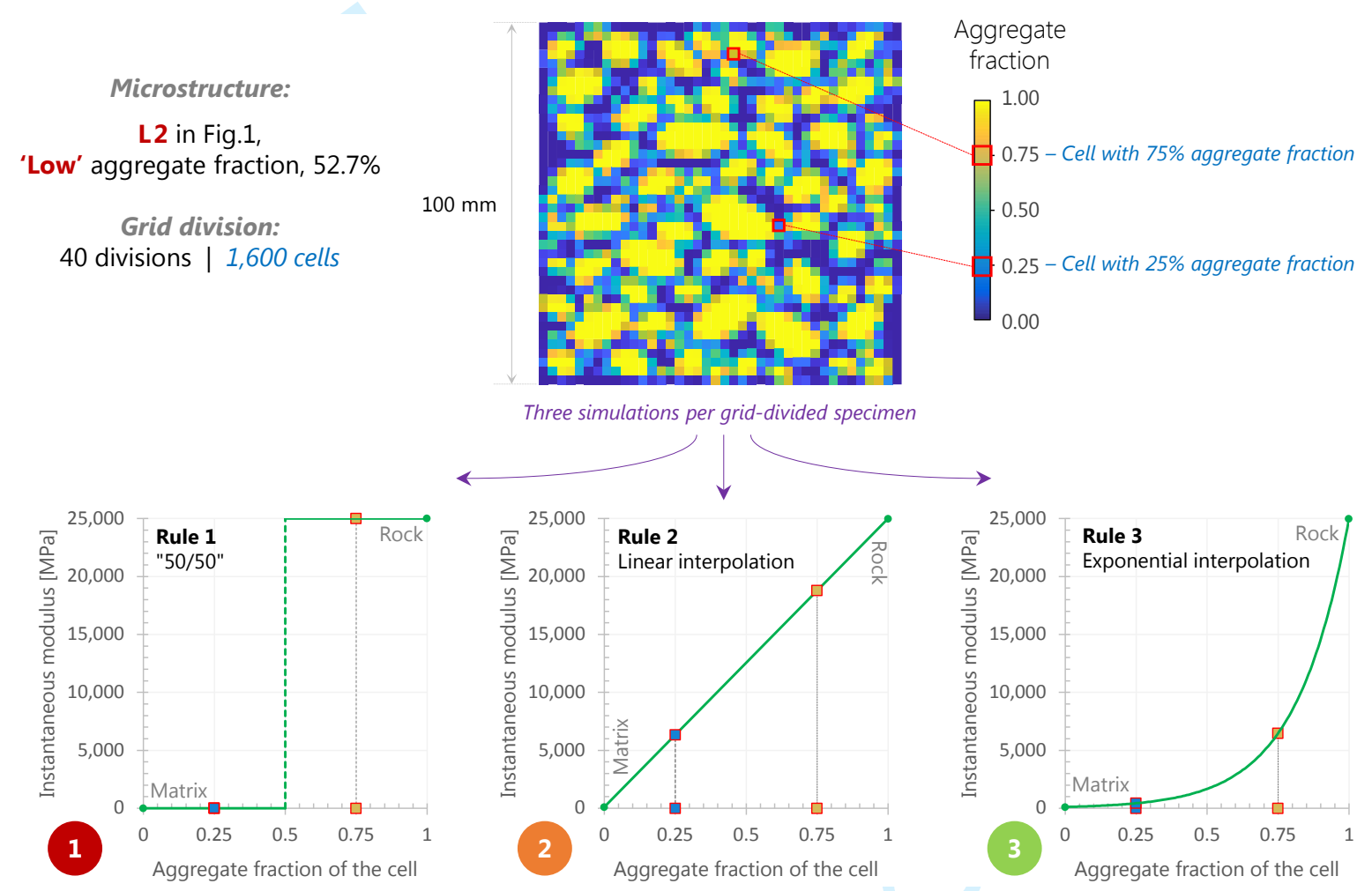

232 Figure 4. Three simple interpolation rules to estimate the mechanical properties of the cells.

234 2.3.1. Rule 1. '50/50'

235 In the first interpolation rule, the mechanical response of a cell is decided using a simple 236 true/false criterion (only two possible outcomes); if the aggregate fraction of the cell is less 237 than $50 \%$, it will behave as pure matrix; on the contrary, if it is over $50 \%$, it will be modelled 238 as pure aggregate. In this way, a cell with $25 \%$ aggregate fraction would behave as pure matrix 239 (viscoelastic, instantaneous modulus $E_{o}=112 \mathrm{MPa}$ ), and a cell with $75 \%$ aggregate fraction 
240 would behave as pure aggregate (elastic, modulus $E=25,000 \mathrm{MPa}$ ). This interpolation rule is

241 considered to be the simplest approximation to the actual response of the microstructure.

\subsubsection{Rule 2. Linear interpolation}

244 A linear interpolation was proposed as the logical step following the aforementioned 245 alternative. In this rule, if the aggregate fraction is not $100 \%$, the cell is modelled linear 246 viscoelastic and its value of instantaneous modulus $E_{o}$ is found by linear interpolation between

247 the $E_{o}$ of the matrix and the Young's modulus of the aggregates, i.e., an intermediate modulus 248 between matrix and aggregates, proportional to the aggregate content of the cell. Only if the 249 aggregate fraction is equal to $100 \%$ would the cell be modelled as pure rock. For instance, a 250 couple of cells with aggregate fraction $25 \%$ and $75 \%$ would be modelled linear viscoelastic, 251 with instantaneous modulus $E_{o} 6,300 \mathrm{MPa}$ and $18,800 \mathrm{MPa}$, respectively.

252 It can probably be foreseen that the grids with few divisions will result in grossly non253 representative responses; however, calculating them is of interest since their results will allow 254 assessing whether the alternatives produce more refined results with increasing divisions, if 255 applicable.

\subsubsection{Rule 3. Exponential interpolation}

258 Finally, an exponential interpolation was proposed as a slightly more refined, yet still extremely 259 simplified approximation to estimating the properties of the cells. The idea of testing an 260 exponential interpolation comes, once again, from the difference in magnitude between 261 aggregates and matrix. From the linear interpolation, it may be noticed that there could be an 262 overestimation of the contribution of the aggregates, since it is more likely that the controlling 263 behavior is viscoelastic (due to the matrix), even at relatively large aggregate fractions. To 264 counteract this effect, the exponential interpolation assigns a lighter 'weight' to the influence 
265 of the aggregates when compared to the linear alternative.

266 A curve of the following shape was used (moduli in MPa):

$$
E_{o}^{c e l l}=E_{o}^{\text {matrix }} e^{k * A G F}
$$

267 where the instantaneous modulus of a cell $\left(E_{o \text { cell }}\right)$ is a function of the modulus of the matrix 268 phase $\left(E_{\text {o matrix }}\right)$, the aggregate fraction of the cell ( $A G F$, ranging from 0 to 1$)$ and a constant $k$ 269 that ensures the fit, i.e. if the aggregate fraction is $100 \%$ then $E_{o}$ cell equals the modulus of the 270 rock. For the moduli used in this study, $k$ was equal to 5.4081. Using the exponential 271 interpolation, for instance, a cell with $25 \%$ aggregate fraction would behave viscoelastic with 272 an instantaneous modulus $E_{o}$ equal to $433 \mathrm{MPa}$ (instead of more than 6,000 MPa in the linear 273 interpolation), and a cell with $75 \%$ aggregate area will have $E_{o}$ equal to $6,468 \mathrm{MPa}$ (instead of $27418,800 \mathrm{MPa}$, linear). If the cell is $100 \%$ aggregate, it will be modelled as pure rock.

\subsection{Conditions for the finite element simulations}

277 As presented in the above sections, six AC microstructures (Figure 1) will be divided using a grid from 1 to 100 divisions, to create 100 grid-divided specimens per microstructure. Also, there are three interpolation rules per grid-divided specimen, which gives a total of 1,800

280 simulations. Six additional simulations are carried out for the microstructures with no grid-like 281 divisions (i.e. traditional meshing of the microstructures in Figure 1), to provide a comparison and contrast with the results of the grid-divided specimens.

The specimens were modelled in FE under a fixed loading scheme. A total displacement of

$2840.1 \mathrm{~mm}$ in monotonic compression was applied during $0.5 \mathrm{sec}$ on a rigid plate tied to the top of 285 the specimens. In the simulations, the vertical movement at the base of the specimens was restricted, and the plate that applied the displacement moved downwards along the vertical axis only, with no rotation.

288 A uniform target mesh size of $0.5 \mathrm{~mm}$ was selected for both the grid-divided specimens and 
289 the six 'original', undivided microstructures. The grid-divided specimens were meshed with 290 rectangular elements (about 40,000), and the microstructures were meshed with triangular 291 elements (around 90,000). Nevertheless, consider that the meshing of the grid-divided 292 specimens is completely neat, since there are no inclusions or irregularities in the geometry 293 (i.e., square elements meshing square subdivisions on a square specimen). Therefore, a model 294 as 'simple' as those presented in Figure 3 could easily be meshed with only a handful FE, 295 greatly reducing its computational cost.

296

\section{3. RESULTS AND ANALYSIS}

298 The analysis of results is presented in three stages. First, the strain in the deformed specimens 299 provides an overview of the differences between the three alternatives. Then, a more detailed 300 analysis of the force-displacement curves gives insight into the accuracy associated with each 301 of the alternatives. Finally, a summary of the force at the end of all the simulations is presented.

\section{$302 \quad 3.1 . \quad$ Strain in the deformed specimens}

303 Figure 5 presents a selection of grid-divided specimens for one microstructure of AC ('L1' 304 from Figure 1) following the three interpolation rules between the mechanical properties of the 305 matrix and the aggregates. The deformations are plotted with a factor of 30 . The grid divisions 306 in Figure 5 correspond to those presented in Figure 3, from 10 divisions upwards.

307 A clear contrast is observed between the alternatives in the specimens with fewer grid 308 divisions ( $\sim 20$ or less). In rule 1 (' $50 / 50$ ') there is a marked difference between the stiff, elastic 309 blocks of rock, and the blocks of matrix, which are very soft and viscous in comparison. The 310 specimens with linear and exponential interpolation exhibit the presence of the aggregates as 311 well, but their overall behavior is more uniform, and the deformations of the vertical (free) 312 edges of the specimens are smaller than in the first alternative. 

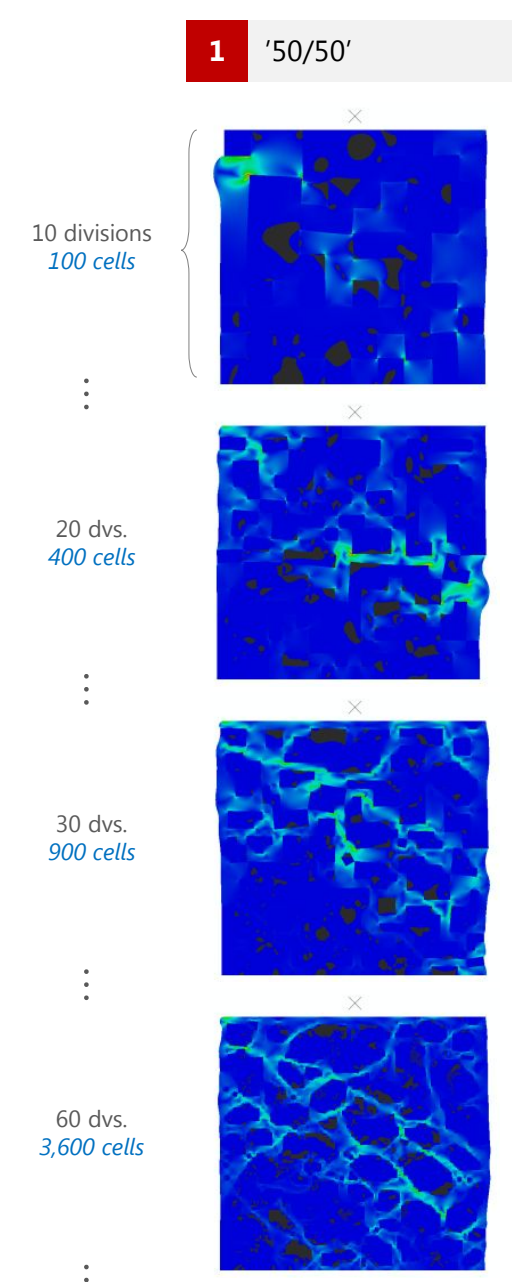

313

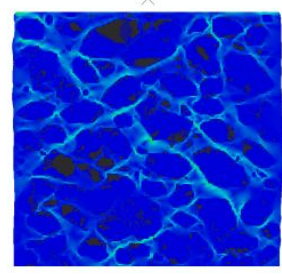

Strain
$\left[\times 10^{-2}\right]$

100 divisions 10,000 cells

Full microstructure

L1 in Figure 1 'Low' aggregate fraction, $52.7 \%$

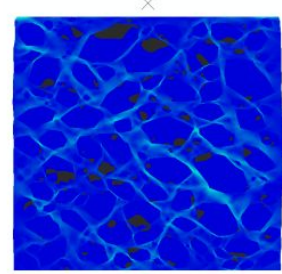

2 Linear interpolation
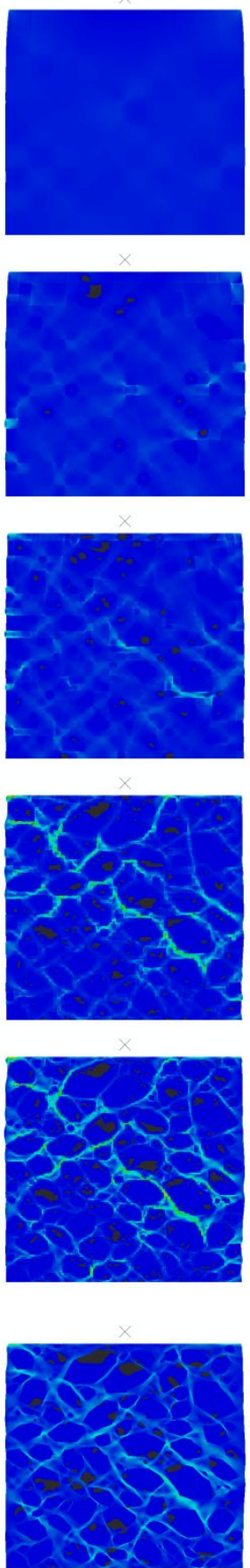

3 Exponential interpolation
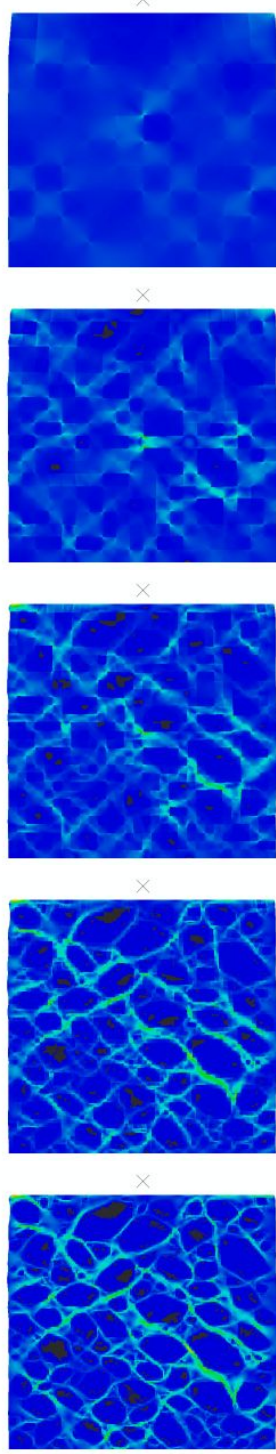

Strain

Strain

$\left[\times 10^{-2}\right]$

$\prod^{3.255}$

$-2.441$

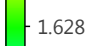

$-0.814$

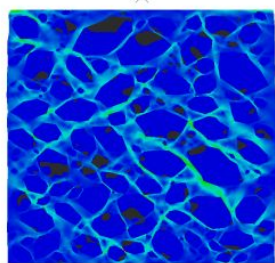

314 Figure 5. Maximum principal strain for selected grid-divided specimens, three interpolation

315 rules. The corresponding microstructure is 'L1' from Figure 1. Notice the difference in the

316 scale among alternatives; results of the full microstructure are identical and differ only in the

317 color scale.

318 
319 When the number of divisions starts to increase, the behavior of the grid-divided specimens 320 becomes more consistent, and similar traits appear among alternatives. The shapes of the 321 aggregates begin to emerge, and strain concentrations gradually converge to similar values; for 322 example, in the three alternatives the strain on the 'paths' between aggregates already has 323 values that are comparable to the microstructural results (lower part of Figure 5 - there is only 324 one microstructure, presented with three color scales for comparison within the alternatives) as 325 early as 30 divisions. In general, by 50-100 subdivisions the actual shapes of the aggregates, 326 the 'paths' of high strain in the matrix and the strain concentrations from the original 327 microstructure are observable in the grid-divided specimens. The improvement in the 328 representation of certain characteristics of the actual behavior is a consequence of the increase 329 in 'resolution,' i.e. the accuracy of the geometric representation of the aggregates.

330 When comparing the results from the grid-divided specimens with the response of the 331 associated microstructure, the three alternatives seem to be converging towards the 332 microstructural response with an increasing number of grid divisions. The convergence is 333 happening at different rates, however; the exponential interpolation appears to offer a closest 334 match with the microstructure 'earlier' (at a lower number of divisions) than the other two 335 alternatives.

336 The methodology used herein produces simplified models in terms of computational cost.

337 Given the conditions in this study, grid-divided specimens were two-dimensional and had less

338 than 50,000 elements. It was possible to (a) create, (b) run and (c) analyze (extract data) from 339 a considerable number of simulations in bulk (1,800), in an estimated cumulative time of 3-4 340 days, using a common office computer. Hence, the development of one simulation would take 341 approximately 3 minutes to run, from start to finish. In contrast, three-dimensional full 342 microstructural models are considerably heavier, usually with more than several hundred 343 thousand finite elements. Each may take more than one day to create and a similar time to run 
344 (Chen et al. 2018, You et al. 2012).

345

$346 \quad 3.2$. Force-displacement curves

347 To find out whether the alternatives offer a reasonable representation of the overall response

348 of the microstructure or not, the analysis continues towards the force-displacement curves of

349 the grid-divided specimens. These curves show the force sustained by the specimen under

350 displacement (in compression) during the total time of the simulation. Figure 6 presents the

351 force-displacement curves of 100 grid-divided specimens of two microstructures, 'L1' and 352 'H1'. 

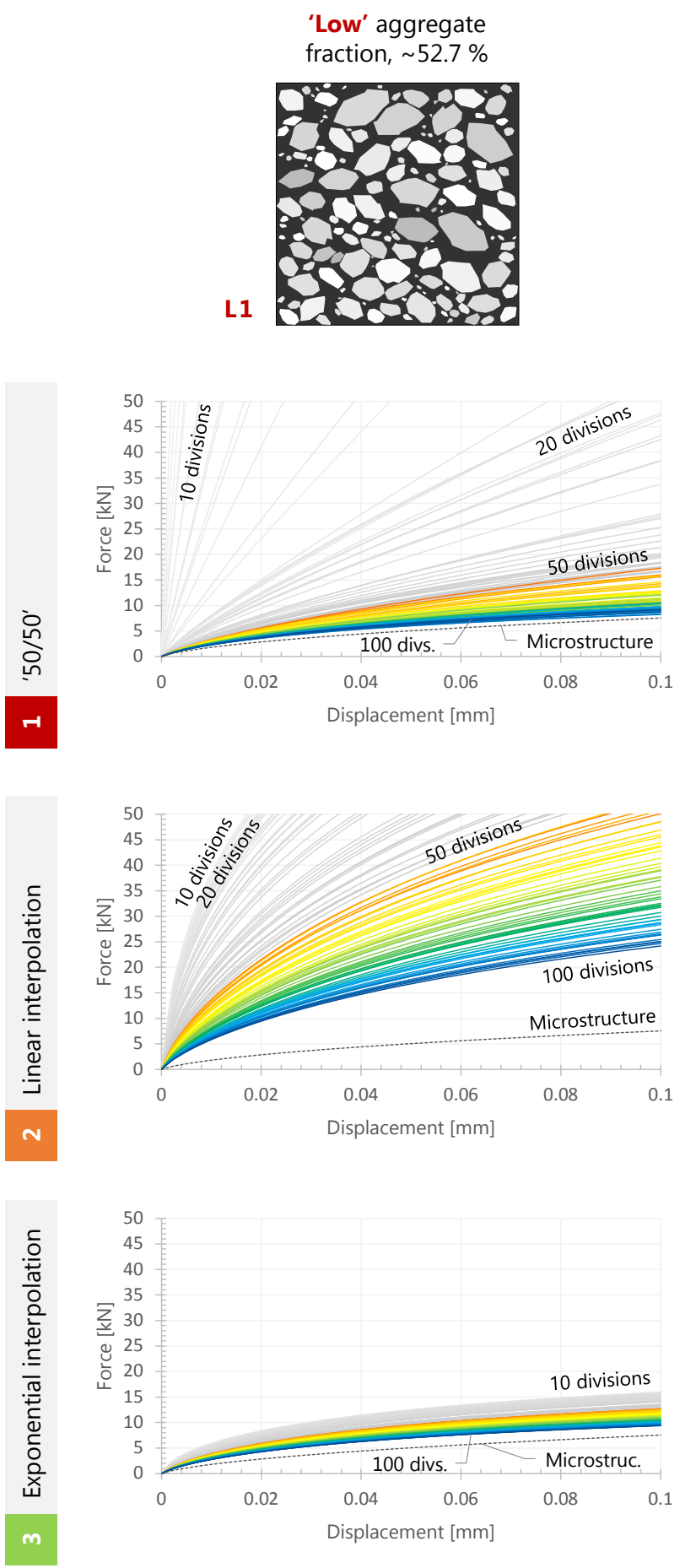

'High' aggregate fraction, $\sim 66.1 \%$

H1
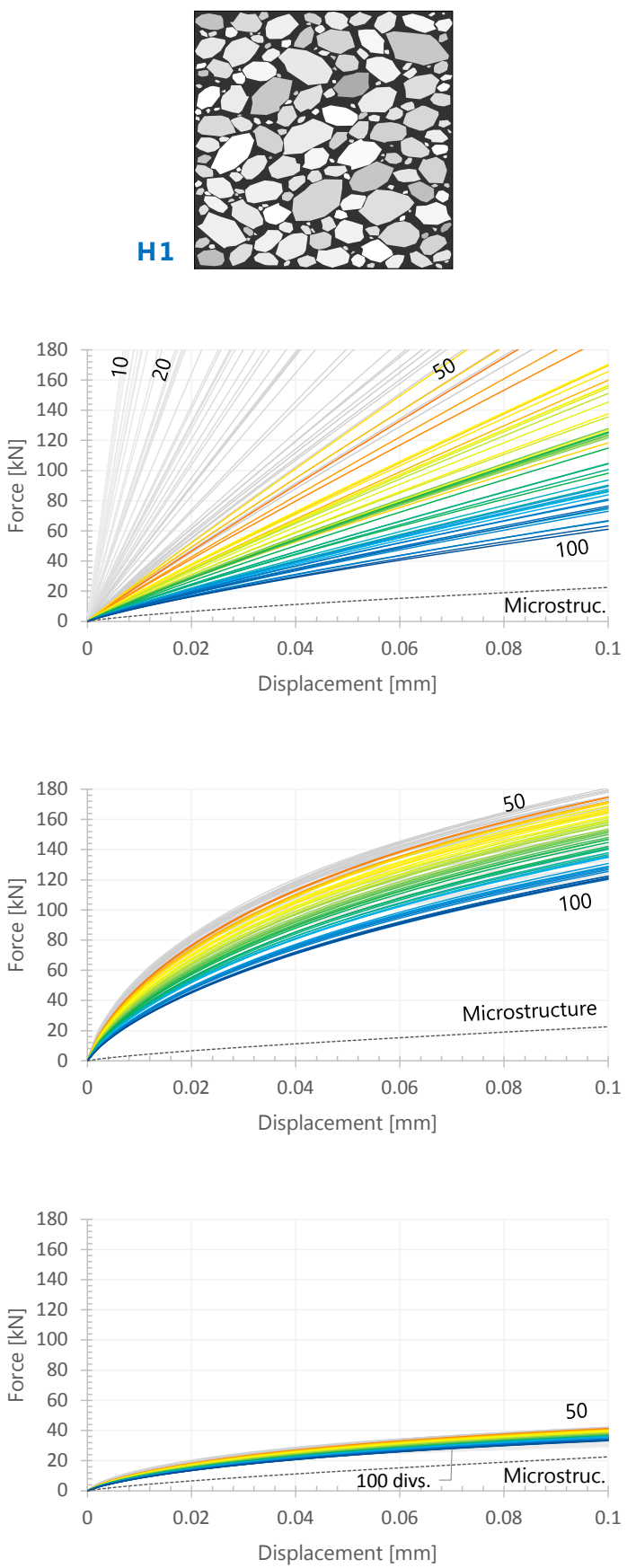

354 Figure 6. Force-displacement curves of 100 grid-divided specimens of two microstructures,

$355 \mathrm{~L} 1$ and $\mathrm{H} 1$, for the three interpolation rules. Curves from 50 to 100 divisions are colored. Notice 356 the difference in vertical scale between specimens. 
359 Clearly, the results of the three interpolations tend to be far from being an accurate 360 representation of the microstructural models. However, an overall consistent behavior is 361 observed, where the three alternatives are converging towards the actual response, and at a 362 medium to high number of divisions, in rules 1 and 3 the force is even within the same order 363 of magnitude as in the original microstructures.

364 It was expected that the 50/50 interpolation rule would grossly overestimate the stiffness of 365 the grid-divided specimens with few divisions/cells, because every cell with medium to high 366 aggregate fraction would behave as pure aggregate. This was particularly true for the 367 microstructures with high aggregate fraction, as it can be observed that the curves converge 368 towards the real response much slowly for the 'H1' grid-divided specimens than for the 'L1'.

369 However, and perhaps surprisingly, at high numbers of divisions/cells the results obtained from 370 the 50/50 interpolation rule are actually better than the linear approach. Initial results with less 371 divisions/cells are rougher, but shortly after 10 divisions (low aggregate fraction) and around 37260 divisions (high aggregate fraction), the force-displacement curves from the 50/50 rule are 373 effectively better approximations to the real response than the linear. At 100 divisions, 50/50 374 results for the low aggregate fraction are even closer to the actual response than the exponential, 375 which offered the best approximation overall. In general terms, while a perfect fit is not obtained, it is interesting to note that such a coarse simplification is still capable, to a point, of 377 representing the results of the original microstructure.

378 While the results from the linear interpolation are more consistent than the ' $50 / 50$ ', they fail 379 to approach the actual response at an acceptable rate. At 100 divisions, this alternative is the 380 farthest of the three from the microstructural results. Interpolating linearly effectively 381 overestimates the contribution of the aggregates to the mechanical response of the cells, 382 misrepresenting the overall behavior of the specimen. While this is corrected as the divisions 383 increase, the contribution of the cells does not increase linearly with aggregate fraction, even 
384 when it does eventually approach the elastic modulus of the rock. On the other hand, the 385 exponential interpolation rule is clearly the better alternative of the three. It converges quickly 386 and is consistently closer to the real response than the other alternatives, at low and high 387 aggregate fractions, even at very low number of divisions/cells. The exponential interpolation 388 is more effectively balancing the mechanical contribution of the cells, and it properly represents 389 the viscoelastic behavior that dominates at low aggregate fractions.

$390 \quad$ Figure 7 presents a summary of the 100-division specimens of the six microstructures, for 391 the three interpolation rules. It can be observed that while the specimens with the same 392 aggregate fraction yield unique results, their behavior is quite consistent (among specimens 393 with the same aggregate fraction); for instance, the curves from microstructures L1, L2 and L3 394 differ in value, but are close together (they have the same aggregate fraction) and differentiated 395 for each of the interpolation rules. 
(a)

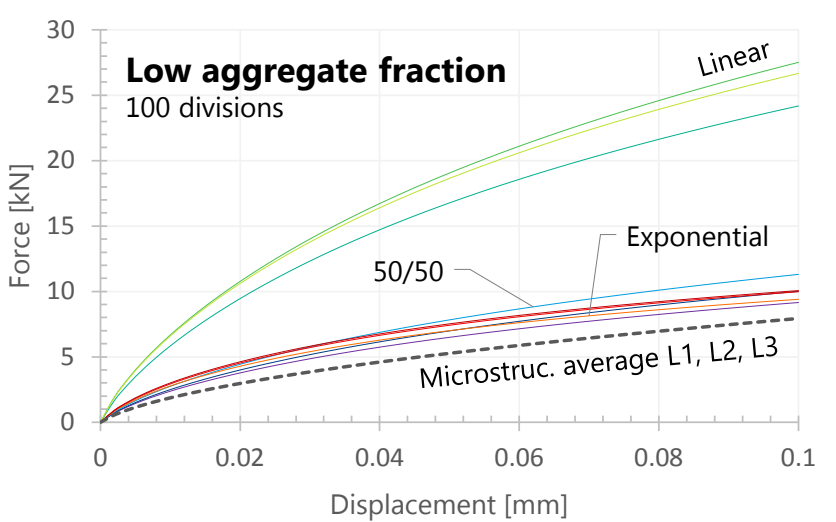

(b)

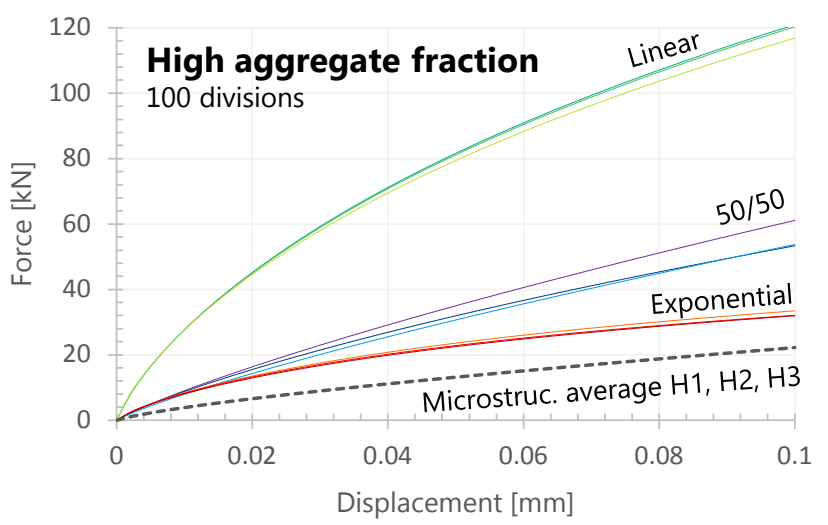

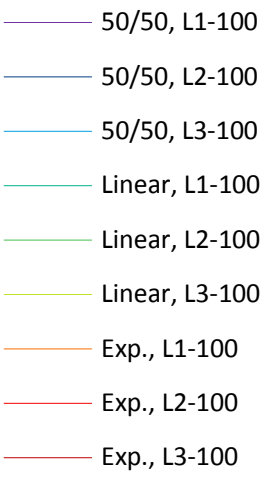

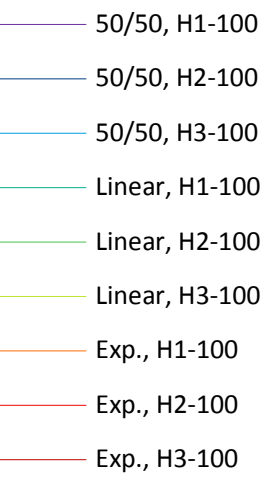

397

398 Figure 7. Force-displacement curves from the grid-divided specimens (100 divisions only) of

399 the six microstructures. Specimens with (a) low aggregate fraction, and (b) high aggregate 400 fraction.

401

$402 \quad 3.3 . \quad$ Force at the end of the simulation

403 An analysis of the force at the end of the simulation offers an interesting insight of the 404 capabilities of the interpolation alternatives. A summary of the force at the end of the simulation 405 for all six microstructures (L1, L2, L3, H1, H2, H3) and grid divisions (1 to 100) is presented 406 in Figure 8. 
(a)

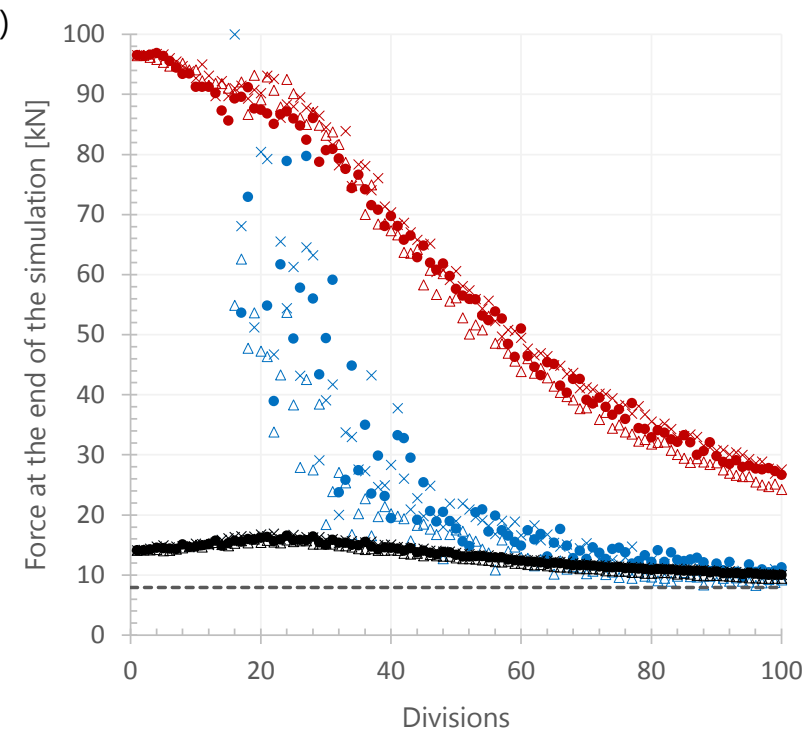

(b)

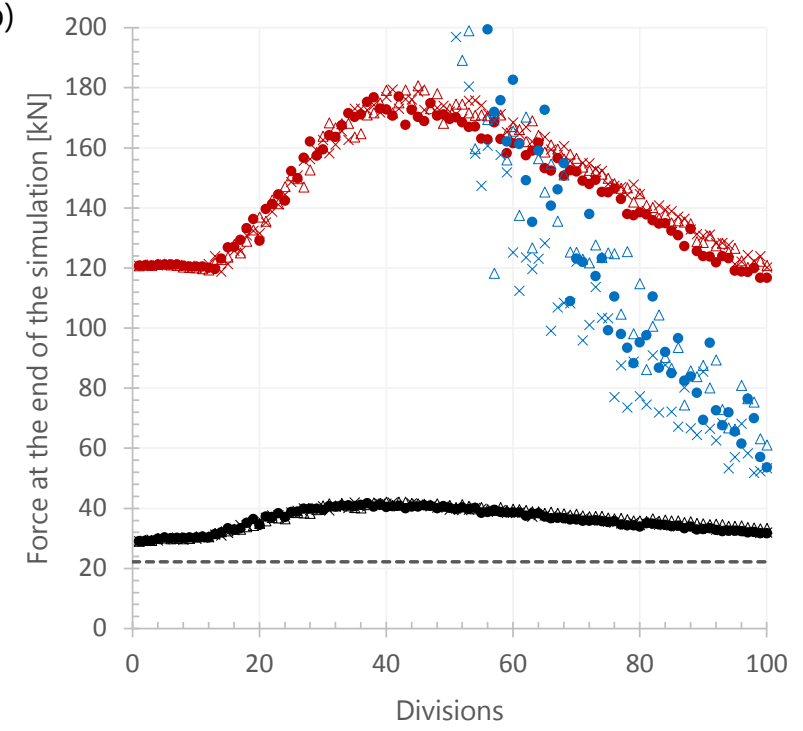

\section{Low aggregate}

fraction, $\mathbf{5 2 . 7 \%}$

$\triangle \quad$ Linear, L1

$\times \quad$ Linear, L2

- Linear, L3

$\triangle \quad 50 / 50$, L1

50/50, L2

- 50/50, L3

$\triangle \quad$ Exp, L1

$\times \quad$ Exp, L2

- $\quad$ Exp, L3

-..... Avg real L1,L2,L3

\section{High aggregate}

fraction, $66.1 \%$

$\triangle \quad$ Linear, $\mathrm{H} 1$

$\times \quad$ Linear, $\mathrm{H} 2$

- Linear, H3

$\triangle \quad 50 / 50, \mathrm{H} 1$

$\times \quad 50 / 50, \mathrm{H} 2$

- 50/50, H3

$\triangle \quad$ Exp, $\mathrm{H} 1$

$\times \quad$ Exp, $\mathrm{H} 2$

- $\operatorname{Exp}, \mathrm{H} 3$

-...-... Avg real $\mathrm{H} 1, \mathrm{H} 2, \mathrm{H} 3$

407

408 Figure 8. Force at the end of the simulation $(\mathrm{t}=0.5 \mathrm{sec}$, displacement $0.1 \mathrm{~mm})$ for all grid-

409 divided specimens; six microstructures (L1-2-3, H1-2-3), three interpolation rules (50/50,

410 Linear, Exp.). (a) Low aggregate area, (b) High aggregate area. Notice the difference in vertical

411 scale.

412

413 Figure 8 provides a clear view of the behavior of the interpolation rules, and the consistency

414 among the simulations. The results from the 50/50 interpolation have a far greater variability,

415 but together with the linear and exponential approximations, their values are consistent among 
416 specimens with the same aggregate fraction (i.e. L1, L2 and L3; H1, H2 and H3). The results 417 are clearly differentiated between interpolation rules, and the nature of the trends is distinct at 418 low and high aggregate fractions, confirming the dominant influence of this parameter in the 419 mechanical response of the specimens. Overall, among the range of divisions selected in this 420 study (i.e. 1 to 100 divisions of a $100-\mathrm{mm}$ side) the alternatives offered a better approximation 421 to the real response for the microstructures with low aggregate fraction; nevertheless, they 422 converge towards the real response for both low and high aggregate fractions, given enough divisions on the grid.

The increased variability among the specimen results for the 50/50 approach may be related 425 to the fact that a step function was used to assign their properties (see Figure 4); i.e. the change between elastic and viscoelastic constitutive behaviors is sudden. Therefore, the response of a 427 cell with $49 \%$ aggregate fraction may differ widely from that of a cell with $51 \%$ aggregate fraction; on the other hand, the transition between these behaviors is smooth for the linear and exponential interpolation rules.

430 An interesting trend appears in the linear and exponential interpolation rules. In the linear 431 alternative, the force of the grid-divided specimens with low aggregate area (Figure 8a), has an apparent bi-linear trend with the number of divisions in the grid; it starts at $96 \mathrm{kN}$ for 1 division, decreases to around $90 \mathrm{kN}$ for 20-25 divisions, and then it reaches a local maximum; it decreases more rapidly after this point. The trend for the exponential rule increases from 1 to $435 \sim 25$ divisions, presents a maximum $(\sim 16 \mathrm{kN})$, and then it decreases, approaching the correct 436 value of the microstructures. A similar trend was clearly observed at high aggregate area 437 (Figure 8b) for both linear and exponential rules; there is a peak in the force sustained by the 438 grid-divided specimens at $\sim 40$ divisions.

439 The results suggest an interesting property of the microstructure, which is able to reach a 440 'maximum potential stiffness' at a certain number (or range) of grid divisions. This value seems 
441 to be dependent on the aggregate fraction of the specimen (higher for increased aggregate

442 fraction), and it is probably related to the ratio of the number of pure aggregate/pure matrix

443 cells in the grid-divided specimen, as well as to the maximum nominal size of the gradation of

444 the microstructures. Additionally, in the exponential alternative at both low and high aggregate

445 fractions, this maximum stiffness was roughly twice the actual response of the microstructure.

446 Results from the 50/50 interpolation rule did not follow this pattern.

447

448

\section{SUMMARY AND CLOSING REMARKS}

449 A simple method was proposed to model the mechanical response of six microstructures of

450 asphalt concrete, three with low and three with high aggregate fraction. This procedure allows

451 for a rough estimate of the actual response of a microstructure, based only on the mechanical 452 properties of its composing phases.

453 The surface of the microstructures was divided into cells using grids with a range of 1 to 454100 divisions (i.e. 1 to 10,000 cells). The aggregate fraction of the cells was used to estimate 455 their mechanical properties, using three simple interpolation rules between the properties of the 456 asphalt matrix and the rock aggregate: (1) 50/50, either matrix or aggregate, (2) linear 457 interpolation, and (3) exponential interpolation.

458 The results were consistent among different microstructures with the same aggregate 459 fraction, validating the use of this parameter as part of the three interpolation rules. While the 460 approximations are less accurate for microstructures with 'high' aggregate fraction, the three 461 alternatives converged towards the original microstructural response with an increasing 462 number of divisions in the grid. Overall, the exponential interpolation rule was clearly the best 463 and closest to the actual response of the microstructure. The '50/50' alternative yielded more 464 variable results, but in some conditions it behaved better than the more 'refined' linear rule 465 (low aggregate fraction, medium to high number of divisions). In fact, results suggest that it is 
466 possible to identify a cell-wise threshold for aggregate fraction (i.e., instead of $50 \%$ in the 50/50

467 interpolation rule) to generate simple interpolation rules that adjust better to the microstructural

468 response. Additionally, the linear and exponential alternatives exhibited a local maximum in 469 the force resisted by the grid-divided specimens, at the same number of divisions (25 divisions 470 for low, 40 for high aggregate fraction).

471 Although the results of this study are an approximation to the real microstructural response, 472 it is remarkable that by using simple interpolation rules between only a couple of points $\left(E_{o}\right.$ of 473 the matrix, and Young's modulus of aggregates) it was possible to approximate a behavior that 474 is inherently highly complex, and that is affected by the heterogeneity of the microstructure. 475 Even though the grid-divided specimens lack a microstructure, to some extent their behavior 476 can approximate that of the actual specimens of asphalt concrete, including characteristics such 477 as strain concentrations and 'paths', overall deformations, and resulting force. Note that at no 478 point during the analysis were bulk AC properties inputted into the models.

479 Potential applications for the methodology presented herein include modelling high volumes 480 of mineral-based materials where micromechanical approaches are not practical due to high 481 computational cost, i.e. full pavement cross-sections. Including microstructural characteristics 482 in such conditions (i.e. aggregates, air voids, and/or cracks and other irregularities in full 483 layers), is currently computationally expensive and may require several millions of finite 484 elements for adequate meshing. The proposed methodology may offer a simplified way to 485 approach these models.

486 The method of grid division is applicable to any multi-phase microstructure, whether 487 acquired by image analysis (real microstructures) or by a packing or otherwise computational 488 algorithm, random or not (virtual microstructures), and the same approach of calculating 489 aggregate fraction in the cells holds in three dimensions. Instead of modulus, any other property 490 may be estimated (e.g. coefficient of thermal or moisture diffusion, with different interpolation 
491 rules) to model a variety of physical processes. Although the methodology is illustrated herein

492 for intact materials, cohesive damage in AC may be considered by replacing the viscoelastic

493 with a damage-encompassing constitutive behavior. Also, as it was mentioned before, the grid

494 does not need to be composed of squares; uneven shapes, or tessellations like Voronoi could

495 be used as well.

496 Accurate computational models of multi-phase materials can easily become 'heavy'; some

497 may take hours or even days to run, using special-purpose servers and supercomputers. In this

498 regard, techniques for 'de-scalation' of complexity such as the one presented in this study may 499 prove useful, enhancing computational efficiency while maintaining reasonable accuracy in the 500 results.

501

502

\section{REFERENCES}

503 Aragão, F.T.S., Kim, Y.R., Lee, J. and Allen, D.H., 2010. "Micromechanical model for 504 heterogeneous asphalt concrete mixtures subjected to fracture failure". Journal of Materials 505 in Civil Engineering, 23(1), pp.30-38.

506 Castillo, D. and Al-Qadi, I.L. 2018. "Importance of heterogeneity in asphalt pavement 507 modeling”. Journal of Engineering Mechanics (ASCE), 144(8): 04018060, 1-13.

508 Castillo, D., Caro, S., Darabi, M.K. and Masad, E. 2015. "Studying the effect of microstructural 509 properties on the mechanical degradation of asphalt mixtures". Construction and Building $510 \quad$ Materials, 93, 70-83.

511 Caro, S., Castillo, D., Darabi, M. and Masad, E., 2018. "Influence of different sources of 512 microstructural heterogeneity on the degradation of asphalt mixtures". International Journal 513 of Pavement Engineering, 19(1), pp.9-23.

514 Chen, J., Wang, H., Dan, H. and Xie, Y., 2018. "Random Modeling of Three-Dimensional 515 Heterogeneous Microstructure of Asphalt Concrete for Mechanical Analysis”. Journal of 
516 Engineering Mechanics, 144(9), 04018083.

517 Darabi, M., Abu Al-Rub, R., Masad, E., Huang, C.W. and Little, D. 2011. “A thermo518 viscoelastic-viscoplastic-viscodamage constitutive model for asphaltic materials". 519 International Journal of Solids and Structures, 48, 191-207.

520 Elseifi, M.A., Al-Qadi, I.L. \& Yoo, P.J., 2006. "Viscoelastic modeling and field validation of 521 flexible pavements”. Journal of Engineering Mechanics, 132, 172-178.

522 Gamez, A., Hernandez, J.A., Ozer, H. and Al-Qadi, I.L., 2018. Development of Domain 523 Analysis for Determining Potential Pavement Damage. Journal of Transportation 524 Engineering, Part B: Pavements, 144(3), p.04018030.

525 Gruber, I., Zinovik, I., Holzer, L., Flisch, A. and Poulikakos, L.D., 2012. "A computational 526 study of the effect of structural anisotropy of porous asphalt on hydraulic conductivity". 527 Construction and Building Materials, 36, 66-77.

528 Hart, D.J. and Wang, H.F., 1995. "Laboratory measurements of a complete set of poroelastic 529 moduli for Berea sandstone and Indiana limestone”. Journal of Geophysical Research: Solid $530 \quad$ Earth, 100(B9), 17741-17751.

531 Hernandez, J., Gamez, A. and Al-Qadi, I.L. 2016. "Effect of wide-base tires on nationwide 532 flexible pavement systems: Numerical modeling”. Transportation Research Record: Journal 533 of the Transportation Research Board, 2590, 104-112.

534 Kim, Y.-R., Allen, D.H. and Little, D.N. 2005. "Damage-Induced Modeling of Asphalt 535 Mixtures through Computational Micromechanics and Cohesive Zone Fracture". Journal of $536 \quad$ Materials in Civil Engineering, 17, 477-484.

537 Kim, Y.R., Lutif, J.S., Bhasin, A. and Little, D.N., 2008. "Evaluation of moisture damage 538 mechanisms and effects of hydrated lime in asphalt mixtures through measurements of 539 mixture component properties and performance testing". Journal of Materials in Civil $540 \quad$ Engineering, 20 (10), 659-667. 
541 Kim, Y., Lee, J. and Lutif, J.E., 2010. "Geometrical evaluation and experimental verification

542 to determine representative volume elements of heterogeneous asphalt mixtures". Journal of 543 Testing and Evaluation, 38 (6), 660-666.

544 Kim, Y.R., Souza, F.V. and Teixeira, J.E.S.L., 2013. "A two-way coupled multiscale model 545 for predicting damage-associated performance of asphaltic roadways". Computational $546 \quad$ Mechanics, 51 (2), 187-201.

547 Masad, E., Muhunthan, B., Shashidhar, N. and Harman, T., 1999. "Internal structure 548 characterization of asphalt concrete using image analysis". Journal of Computing in Civil $549 \quad$ Engineering, 13 (2), 88-95.

550 Obaidat, M.T., Ghuzlan, K.A. and Alawneh, M.M., 2017. "Analysis of volumetric properties 551 of bituminous mixtures using cellular phones and image processing techniques". Canadian 552 Journal of Civil Engineering, 44 (9), 715-726.

553 Ozer, H., Ghauch, Z.G., Dhasmana, H. and Al-Qadi, I.L., 2016. "Computational 554 micromechanical analysis of the representative volume element of bituminous composite 555 materials". Mechanics of Time-Dependent Materials, 20 (3), 441-453.

556 Rahmani, E., Darabi, M.K., Little, D.N. and Masad, E.A., 2017. Constitutive modeling of 557 coupled aging-viscoelastic response of asphalt concrete. Construction and Building Materials, $558 \quad 131,1-15$.

559 Shakiba, M., Darabi, M.K., Abu Al-Rub, R.K., You, T., Little, D.N. and Masad, E.A., 2015.

560 "Three-dimensional microstructural modelling of coupled moisture-mechanical response of 561 asphalt concrete”. International Journal of Pavement Engineering, 16(5), 445-466.

562 Underwood, B.S. and Kim, Y.R., 2011. "Experimental investigation into the multiscale 563 behaviour of asphalt concrete". International Journal of Pavement Engineering, 12 (4), 357 564370.

565 Wang, H. and Al-Qadi, I.L., 2008. Comparison between mechanistic analysis and in-situ 
566 response of full-depth flexible pavements. In Airfield and Highway Pavements: Efficient 567 Pavements Supporting Transportation's Future, 1-15.

568 Wang, H. and Al-Qadi, I.L., 2009. Combined effect of moving wheel loading and three569 dimensional contact stresses on perpetual pavement responses. Transportation Research $570 \quad$ Record, $2095(1), 53-61$.

571 Witthüser, K., Arnepalli, D. and Singh, D.N., 2006. "Investigations on diffusion characteristics 572 of granite and chalk rock mass". Geotechnical \& Geological Engineering, 24 (2), 325-334.

573 Yasar, E. and Erdogan, Y., 2004. "Correlating sound velocity with the density, compressive 574 strength and Young's modulus of carbonate rocks”. International Journal of Rock Mechanics 575 and Mining Sciences, 41(5), 871-875.

576 Yokoyama, T. and Nakashima, S., 2005. "Diffusivity anisotropy in a rhyolite and its relation 577 to pore structure". Engineering Geology, 80 (3-4), 328-335.

578 You, Z., Adhikari, S., and Kutay, E., 2009. "Dynamic modulus simulation of the asphalt 579 concrete using the X-ray computed tomography images”. Materials and Structures, 42, 617$580 \quad 630$.

581 You, T., Abu Al-Rub, R., Darabi, M.K., Masad, E. and Little, D. 2012. "Three-dimensional 582 microstructural modeling of asphalt concrete using a unified viscoelastic-viscoplastic583 viscodamage model”. Construction and Building Materials, 28, 531-548.

584 Zelelew, H. and Papagiannakis, A.T., 2011. "A volumetrics thresholding algorithm for 585 processing asphalt concrete X-ray CT images". International Journal of Pavement $586 \quad$ Engineering, $12(6), 543-551$. 